\title{
Biomicroscopia ultrassônica na viscocanalostomia
}

\author{
Ultrasound biomicroscopy in viscocanalostomy
}

Lígia Maria Fernandes Ribeiro', Dália Maria Martins Meira², Eduardo Marinho Saraiva ${ }^{1}$,Joaquim Fernando de Oliveira Sequeira ${ }^{3}$, Luís Manuel de Sousa Pinto Agrelos ${ }^{4}$

\section{Resumo}

Objetivo: Estudar por biomicroscopia ultrassônica (UBM) de alta frequência as características anatômicas da viscocanalostomia, e a relação dessas características com a redução da pressão intraocular. Métodos: Estudo transversal, que incluiu nove olhos (sete pacientes) submetidos à viscocanalostomia, e posteriormente examinados por UBM de alta frequência $(80 \mathrm{MHz})$. Os parâmetros da UBM avaliados após um follow-up mínimo de seis meses foram: presença de espaço intraescleral, comprimento e altura máximos do espaço intraescleral, e a espessura mínima da membrana trabéculo-descemética (MTD) residual. O sucesso cirúrgico definiu-se como pressão intraocular $(\mathrm{PIO})<22 \mathrm{mmHg}$ ou redução de $20 \%$ da PIO sem medicação tópica. Possíveis associações entre as variáveis da UBM e o resultado cirúrgico foram avaliados. Resultados: $\mathrm{O}$ tempo médio entre a cirurgia e a realização da UBM foi de 15,5 $\pm 8,8$ meses (6 - 29 meses). Verificou-se uma redução da PIO de 23,5 $\pm 6,9 \mathrm{mmHg}(13,7-32,0)$ pré-operatória para $14,5 \pm 2,4 \mathrm{mmHg}(10,7-17,3)$ pós-operatória $(\mathrm{p}<0,05)$. Identificou-se a presença de espaço intraescleral em todos os olhos. A média do comprimento máximo do espaço intraescleral era $1,83 \pm 0,51 \mathrm{~mm}$; a média da altura máxima do espaço intraescleral era $0,36 \pm$ $0,17 \mathrm{~mm}$; e a média da espessura mínima da MTD era $0,14 \pm 0,07 \mathrm{~mm}$. Não foram encontradas correlações significativas entre o valor da PIO pós-operatória e o comprimento do espaço intraescleral $\left(\mathrm{r}^{2}=0,359\right)$, a altura do espaço intraescleral $\left(\mathrm{r}^{2}=0,017\right)$ e a espessura da MTD $\left(\mathrm{r}^{2}=0,003\right)$. Conclusão: Em pacientes submetidos à viscocanalostomia, a UBM após um follow-up mínimo de seis meses identificou o espaço intraescleral em todos os olhos. Não se encontrou qualquer correlação estatisticamente significativa entre os valores de PIO pós-operatória e as características anatômicas do espaço intraescleral.

Descritores: Esclera/ultrassonografia; Lâmina limitante posterior da córnea/ultrassonografia; Pressão intraocular; Cirurgia filtrante/métodos; Glaucoma de ângulo aberto/cirurgia; Hipertensão ocular/cirurgia

\section{ABSTRACT}

Purpose: To evaluate by high frequency ultrasound biomicroscopy (UBM) the anatomical characteristics of viscocanalostomy and their relationship with the intraocular pressure (IOP) lowering. Methods: A transversal study which included 9 eyes (7 patients) undergoing viscocanalostomy and examined by high frequency UBM $(80 \mathrm{MHz})$. Several UBM variables were evaluated, including the presence of the intrascleral space, the maximum length and height of the intrascleral space and the minimum thickness of residual trabeculo-Descemet membrane (TDM). Surgical success was considered to be achieved when IOP was $<22 \mathrm{mmHg}$ or the IOP was lowered $20 \%$ or more without the use of any medication. The possible association between UBM variables and the surgical outcome was determined. Results: The mean time between surgery and the UBM examination was $15.5 \pm 8.8$ months (range 6-29). The mean IOP decreased from a preoperative value of $23.5 \pm 6.9 \mathrm{~mm} \mathrm{Hg}$ (range 13.7-32.0) to $14.5 \pm 2.4 \mathrm{mmHg}$ (range 10.7-17.3) postoperative $(p<0.05)$. The presence of an intrascleral space was a constant finding. The mean length of the intrascleral space was $1.83 \pm 0.51 \mathrm{~mm}$, the mean height was $0,36 \pm 0,17 \mathrm{~mm}$; and the mean TDM thickness was $0.14 \pm 0.07 \mathrm{~mm}$. There were a poor correlation between the level of IOP at the time of UBM and the lenght of the intrascleral space $\left(r^{2}=0.359\right)$, the height of the intrascleral space $\left(r^{2}=0.017\right)$ or the thickness of the remaining TDM ( $\left.r^{2}=0.003\right)$. Conclusion: In patients undergoing viscocanalostomy, UBM examination after a minimum of 6-month follow-up period showed the presence of an intrascleral space in all patients. There was no statiscally significant relationship between the level of IOP and the anatomical characteristics of the intrascleral space.

Keywords: Sclera/ultrasonography; Descemet membrane/ultrasonography

Intraocular pressure; Filtering surgery/methods; Glaucoma, open-angle/surgery; Ocular hypertension/surgery

\footnotetext{
${ }^{1}$ Interno do Internato complementar de Oftalmologia do Centro Hospitalar Vila Nova de Gaia - Espinho - EPE - Portugal;

${ }^{2}$ Centro Hospitalar Vila Nova de Gaia - Espinho - EPE - Portugal;

${ }^{3}$ Centro Hospitalar Vila Nova de Gaia -Espinho - EPE - Portugal;

${ }^{4}$ Centro Hospitalar Vila Nova de Gaia -Espinho - Centro Hospitalar Vila Nova de Gaia - Espinho - EPE - Portugal.

Serviço Oftalmologia, Centro Hospitalar Vila Nova de Gaia - Espinho - EPE - Portugal

Os autores declaram inexistir conflitos de interesse

Recebido para publicação em 12/7/2011 - Aceito para publicação em 19/11/2011
} 


\section{INTRODUÇÃO}

A cirurgia não penetrante de glaucoma ( $\mathrm{CNPG})$, especificamente a técnica de esclerotomia profunda, foi introduzida na década de 80 por Fyodorov $^{(1)} \mathrm{e} \operatorname{Kozlov}^{(2)}$. A principal vantagem das CNPG reside no fato de evitar a comunicação direta entre a câmara anterior e os espaços subconjuntivais, diminuindo assim potenciais complicações cirúrgicas pós-operatórias nomeadamente $\mathrm{o}$ excesso de filtração que conduz a hipotonia/atalamia e descolamento coroideo. Estudos retrospectivos e prospectivos que comparam a clássica trabeculectomia com as técnicas cirúrgicas de CNPG, apontam para taxas de sucesso cirúrgico semelhantes ${ }^{(3,4)}$.

Em 1989, Stegmann ${ }^{(5)}$ modificou o procedimento da esclerotomia profunda, injetando material viscoelástico no canal de Schlemm com o objetivo de dilatar o canal e seus eferentes de forma a facilitar a drenagem do humor aquoso. O procedimento desenvolvido por Stegmann, chamado viscocanalostomia, surgiu na tentativa de combater as elevadas taxas de complicações e falência da trabeculectomia (cirurgia penetrante) na população africana.

O objetivo da viscocanalostomia é obter uma área de filtração que substituirá a malha trabecular e o tecido trabecular justacanalicular não funcionantes. Este procedimento cirúrgico aumenta a drenagem do humor aquoso ao criar um espaço/reservatório intraescleral, que está ligado ao canal de Schlemm permeável. O mecanismo exato da drenagem do humor aquoso, e consequente diminuição da PIO, na viscocanalostomia é controverso. Teoricamente, vários mecanismos podem estar envolvidos, nomeadamente (1) filtração subconjuntival permanente (semelhante à trabeculectomia); (2) drenagem para o sistema canalicular que escoa para a circulação venosa e/ou espaço uveoescleral, com ou sem um lago intraescleral associado; e (3) drenagem do canal de Schlemm para capilares e veias dos canais intraesclerais e tecido subconjuntival.

A avaliação do segmento anterior com biomicroscopia ultrassônica (UBM) permite a visualização in vivo de pormenores da câmara anterior, do corpo ciliar e do tecido límbico córneoescleral. Estudos prévios com UBM sugerem que este exame pode contribuir para a compreensão do mecanismo subjacente à redução da PIO após a CGNP, uma vez que permite visualizar a vesícula de filtração subconjuntival, o espaço intraescleral e a membrana trabéculo-descemética ${ }^{(6-11)}$.

O presente trabalho tem como objetivo estudar por UBM de alta frequência $(80 \mathrm{MHz})$ as características anatômicas dos tecidos do segmento anterior após viscocanalostomia, e a relação dessas características com a respectiva redução da PIO, na tentativa de identificar os parâmetros associados ao sucesso cirúrgico.

\section{Métodos}

Estudo transversal, que incluiu nove olhos de sete pacientes submetidos à viscocanalostomia. Os mecanismos de redução da PIO foram investigados através da exploração da área cirúrgica por UBM. Todos os pacientes incluídos no estudo tinham glaucoma não controlado apesar de terapêutica médica máxima tolerada, e não apresentavam história prévia de tratamento a laser ou cirurgia de glaucoma. Dos nove olhos, cinco apresentavam glaucoma primário de ângulo aberto (GPAA) e quatro, glaucoma pseudoesfoliativo (GPEX).

Todas as cirurgias foram realizadas pelo mesmo cirurgião (DM) segundo a técnica descrita por Stegmann ${ }^{(5)}$.

O exame oftalmológico nas consultas de followup incluiu melhor acuidade visual corrigida, medida da PIO com tonômetro de aplanação de Goldmann, biomicroscopia do segmento anterior e fundoscopia.

Foram incluídos apenas olhos com sucesso cirúrgico, definido como pressão intraocular (PIO) $<22 \mathrm{mmHg}$ ou redução de $20 \%$ da PIO sem medicação tópica.

\section{Viscocanalostomia - técnica cirúrgica}

A intervenção começa com a dissecção da conjuntiva e da Tenon, com base fórnix. Em seguida, realiza-se um retalho escleral superficial, com $5 \times 5 \mathrm{~mm}$ e aproximadamente $1 / 3$ da espessura escleral, que se estende até $1-2 \mathrm{~mm}$ de córnea clara. Um segundo retalho escleral triangular mais profundo é dissecado e excisado, permitindo a abertura do canal Schlemm e a exposição da membrana de Descemet. As extremidades proximais do canal de Schlemm são canalizadas com uma cânula de Grieshaber, e subsequentemente as extremidades do canal de Schlemm e o espaço intraescleral são preenchidos com ácido hialurônico de alta viscosidade. O retalho superficial é suturado de forma apertada com nylon 10-0 para que o humor aquoso permaneça no espaço intraescleral e não atinja o espaço subconjuntival, evitando a formação de vesícula de filtração. Injeta-se ácido hialurônico de alta viscosidade sob o retalho superficial para prevenir adesão imediata do retalho ao leito escleral. A conjuntiva é suturada com fio absorvível (Vycril) 7-0.

\section{Exame UBM}

O iUltraSound UBM (Ophthalmic Imaging System, iScience 2007) foi usado para obter imagens de alta frequência $(80 \mathrm{MHz})$, permitindo uma resolução axial de $25 \mu \mathrm{m}$. Os doentes foram examinados na posição de decúbito dorsal, e aplicou-se um gel de baixa viscosidade na área cirúrgica antes do scan ecográfico. Os parâmetros da UBM avaliados foram: presença de espaço intraescleral, comprimento e altura máximos do espaço intraescleral, e a espessura mínima da membrana trabéculo-descemética (MTD) residual. O exame de UBM foi realizado sempre pelo mesmo oftalmologista (DM).

\section{Análise estatística}

A análise estatística foi realizada usando SPSS para Windows versão 17.0. Os valores médios dos diferentes parâmetros foram comparados usando o teste t-Student (va- 

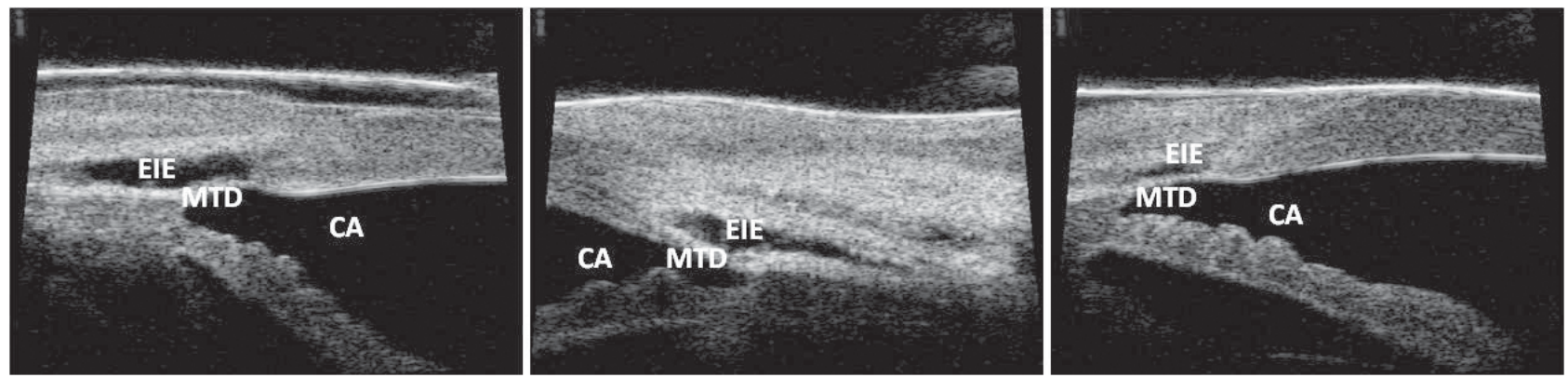

Figura 1: Imagens de UBM de 3 doentes submetidos à viscocanalostomia; CA - câmara anterior; MTD - membrana trabéculo-descemética; EIE - espaço intraescleral
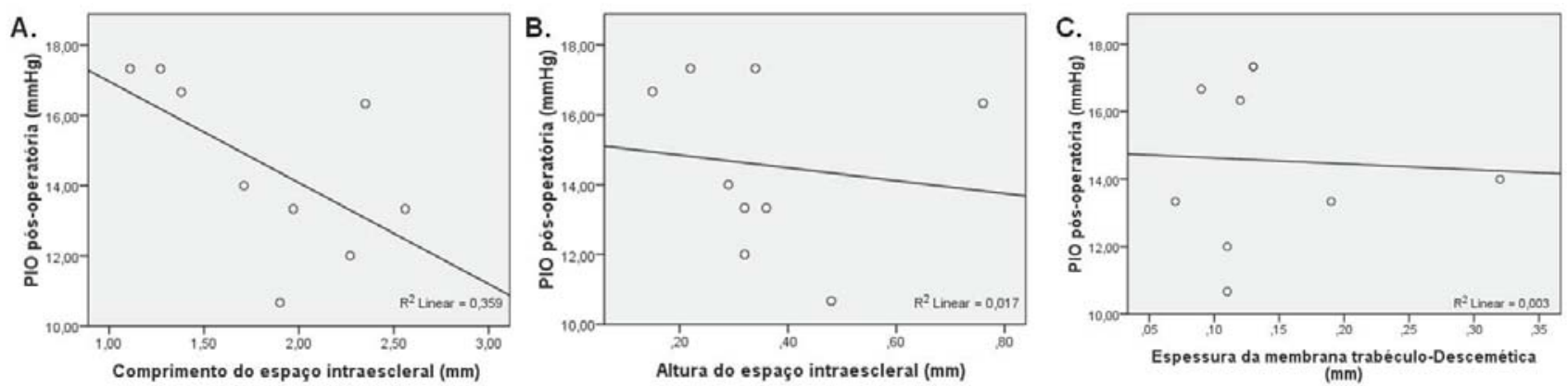

Gráficos 1: (A) - Correlação entre PIO e comprimento do espaço intraescleral ( $\left.\mathrm{r}^{2}=0,359\right)$; (B) - correlação entre PIO e altura do espaço intraescleral $\left(\mathrm{r}^{2}=0,017\right) ;(\mathrm{C})$ - correlação entre PIO e espessura da membrana trabéculo-descemética $\left(\mathrm{r}^{2}=0,003\right)$
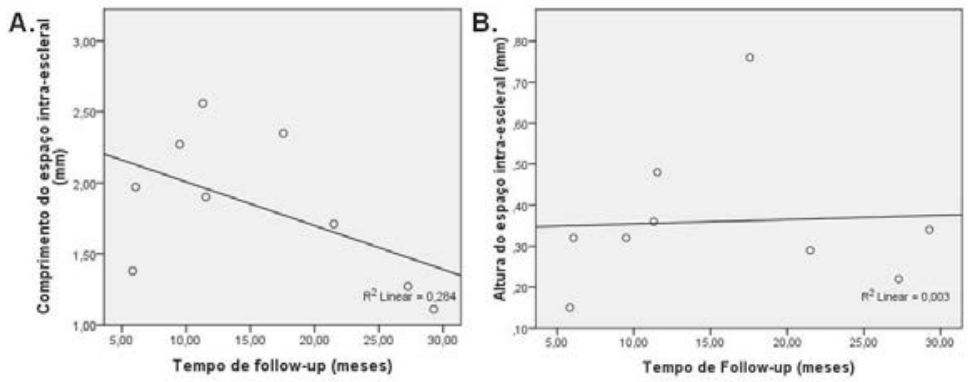

Gráficos 2: (A) - correlação entre comprimento do espaço intraescleral e tempo de follow-up $\left(\mathrm{r}^{2}=0,284\right)$; (B) correlação entre comprimento do espaço intraescleral e tempo de follow-up $\left(\mathrm{r}^{2}=0,003\right)$

lores de $\mathrm{p}<0,05$ foram considerados estatisticamente significativos). A análise de regressão linear foi usada para estudar a relação entre PIO, comprimento e altura do espaço intraescleral, espessura da MTD e tempo de follow-up.

\section{$\underline{\text { RESULTADOS }}$}

Todos os pacientes incluídos neste estudo $(\mathrm{n}=7)$ eram de raça caucasiana, com uma média etária de 67,9 $\pm 11,1$ anos (50-83 anos). Cinco (71,4\%) pacientes eram do sexo masculino e dois $(28,6 \%)$ do sexo feminino. O tempo médio entre a cirurgia e a data da última consulta de oftalmologia, em que se realizou a UBM, foi de 15,5 \pm 8,8 meses (6-29 meses).
Clinicamente, três $(33 \%)$ olhos apresentavam vesícula de filtração subconjuntival.

À data da última consulta de follow-up, oito olhos apresentavam $\mathrm{PIO}<21 \mathrm{mmHg}$ sem medicação tópica e um olho com redução de $20 \%$ da PIO sem medicação tópica, mas com necessidade de combinar dois fármacos tópicos para controle tensional, quando pré-operatoriamente fazia uso de quatro fármacos. Nenhum paciente foi submetido à goniopunctura com laser neodymium:YAG (Nd:YAG).

\section{Pressão intraocular}

Verificou-se uma redução da PIO de 23,5 \pm 6,9 $\mathrm{mmHg}(13,7-32,0)$ pré-operatória para $14,5 \pm 2,4$ 


\section{Tabela 1}

Valores médios dos parâmetros da UBM avaliados

\begin{tabular}{ll}
\hline Parâmetro $(\mathrm{n}=9)$ & Média \pm DP $(\mathbf{m a ́ x}-\mathbf{m i n})(\mathrm{mm})$ \\
\hline Comprimento máximo do espaço intraescleral & $1.83 \pm 0.51(1.11-2.56)$ \\
Altura máxima do espaço intraescleral & $0.36 \pm 0.17(0.15-0.76)$ \\
Espessura da membrana trabéculo-descemética & $0.14 \pm 0.07(0.07-0.32)$ \\
\hline
\end{tabular}

mmHg $(10,7-17,3)$ na última consulta pós-operatória $(\mathrm{p}<0,01)$. A média do número de fármacos usados antes da cirurgia era $2,78 \pm 0,7$, versus $0,22 \pm 0,7$ na última consulta de follow-up $(\mathrm{p}<0,05)$. Apenas um doente necessitava de fazer medicação tópica com 2 fármacos para controle da PIO; os restantes doentes estavam controlados sem medicação.

\section{Biomicroscopia ultrassônica}

A UBM identificou a presença de espaço intraescleral, hipoecogênico e bem circunscrito, em todos os pacientes; bem como uma linha hiperreflectiva que se estende desde a córnea periférica até ao esporão escleral, interposta entre a câmara anterior e o espaço escleral (Figura 1).

A média do comprimento máximo do espaço intraescleral era $1,83 \pm 0,51 \mathrm{~mm}$; a média da altura máxima do espaço intraescleral era $0,36 \pm 0,17 \mathrm{~mm}$; e a média da espessura mínima da MTD era 0,14 $\pm 0,07 \mathrm{~mm}$ (Tabela 1).

As relações entre o valor da PIO pós-operatória e o comprimento do espaço intraescleral $\left(\mathrm{r}^{2}=0,359\right)$, a altura do espaço intraescleral $\left(\mathrm{r}^{2}=0,017\right)$ e a espessura da MTD $\left(\mathrm{r}^{2}=0,003\right)$ são fracas, e não apresentam significado estatístico (Gráficos $1 \mathrm{~A}$ a $\mathrm{C}$ ). A relação entre o tempo de follow-up e o comprimento do espaço intraescleral $\left(\mathrm{r}^{2}=0,284\right)$, e a altura do espaço intraescleral $\left(\mathrm{r}^{2}=0,003\right)$ também são fracas e estatisticamente sem significado (Gráficos 2 A e B).

\section{Discussão}

A UBM é um método não invasivo, de contato, que permite avaliar a morfologia e as alterações estruturais produzidas pelas cirurgias de glaucoma. Neste estudo, é usado pela primeira vez um UBM de elevada frequência $(80 \mathrm{MHz})$ e resolução axial de $25 \mu \mathrm{m}$ para estudar a área cirúrgica após-viscanalostomia. Estudos prévios utilizaram UBM com uma frequência de $50 \mathrm{MHz}$ e resolução axial de $50 \mu \mathrm{m}^{(6-8,10,11)}$.

Num olho normal, o local anatômico de maior resistência à drenagem do humor aquoso é o tecido conjuntivo justacanalicular da malha trabecular. A viscocanalostomia faz um bypass a esta área, porque é criada uma membrana suficientemente permeável à passagem do humor aquoso da câmara anterior para o espaço escleral. Aliás, no ato cirúrgico observa-se o hu- mor aquoso a repassar a MTD. No presente estudo, e de acordo com um estudo elaborado por Negri-Aranguren et al. ${ }^{(6)}$, o exame com UBM permitiu a observação em todos os doentes, duma linha fina hiperrefletiva, que se estende da periferia da córnea até ao esporão escleral, e que corresponde à MTD dissecada.

Outro achado constante nas imagens de UBM, foi a presença de uma área não refletiva contígua com a linha anteriormente descrita, correspondendo ao espaço intraescleral preenchido por humor aquoso.

Negri-Aranguren et al. ${ }^{(6)}$ num estudo de UBM após viscocanalostomia encontraram o espaço intraescleral em todos os doentes $(n=23)$ no $4^{\circ}$ mês após a cirurgia e em apenas $50 \%$ no $13^{\circ}$ mês. Roters et al. ${ }^{(7)}$, em estudo semelhante detectaram o espaço intraescleral em $86,6 \%$ dos casos um mês após a cirurgia, usando um UBM com uma resolução axial de $50 \mu \mathrm{m}$. No nosso estudo foi possível detectar a persistência deste espaço em todos os olhos, mesmo após seis meses de pós-operatório, o que pode ser justificado pela maior capacidade de resolução do UBM $(25 \mu \mathrm{m})$ comparativamente aos estudos previamente mencionados. A presença deste espaço sugere um fluxo contínuo de fluído que passa através da MTD, e subsequentemente é drenado.

Roters et al..$^{(7)}$ concluiram que a MTD era estável quando a sua espessura era inferior a $0,13( \pm 0,02) \mathrm{mm}$. Este dado está de acordo com o nosso trabalho, em que a MTD apresentava uma espessura inferior a $0,15 \mathrm{~mm}$ em oito dos nove olhos.

O canal de Schlemm não foi visualizado em nenhum paciente, apesar do transdutor da UBM ser de $80 \mathrm{MHz}$, o que muito provavelmente se justificará pelo tempo mínimo de realização da UBM ser de seis meses. Seria interessante comparar com a capacidade do iUltraSound UBM visualizar o canal de Schlemm no pós-operatório imediato de viscocanalostomia.

Em concordância com estudo prévios ${ }^{(7,10)}$, no nosso estudo não se encontrou nenhuma relação entre os dados funcionais (redução da PIO) e os dados anatômicos avaliados por UBM (dimensões do espaço intraescleral e/ou espessura da MTD). Apenas Marchini et al. ${ }^{(8)}$ encontraram na avaliação pós-operatória da esclerotomia profunda com implantes de ácido hialurónico por UBM, correlação significativa entre a a redução da PIO e a presença de espaço intraescleral, refletividade da bolha conjuntival e uma área hipoecóica supraciliar.

Park et al. ${ }^{(9)}$ estudaram por UBM as alterações 
anatômicas no local da esclerotomia após cirurgia combinada de viscocanalostomia com cirurgia de catarata. O espaço intraescleral estava presente em $66,7 \%$ dos casos aos 12 meses, mas observaram uma diminuição significativa da área, do comprimento longitudinal e da altura entre três e 12 meses. No nosso estudo, não se verificaram variações significativas no lago escleral com o aumento do tempo de follow-up. No entanto, este dado tem que ser analisado com cautela porque se trata de um estudo transversal e não prospectivo.

A incidência de vesícula subconjuntival de filtração na viscocanalostomia é controversa, e varia entre 0 e $93 \%$ nos estudos publicados. No nosso estudo, a vesícula subconjuntival é visualizada em $33 \%$ dos olhos, mas não foi possível detectar por UBM uma via bem definida que una o lago intraescleral à vesícula subconjuntival.

O nosso estudo tem várias limitações, nomeadamente ser um estudo transversal com uma amostra de tamanho reduzido. No entanto, os nossos resultados sugerem que o sucesso cirúrgico relaciona-se com a presença dum espaço intraescleral patente, mas não parece ter uma relação directa com as dimensões desse espaço.

\section{RefERÊNCIAS}

1. Fyodorov SN. Non-penetrating deep sclerectomy in openangle glaucoma. Eye Microsurg. 1989;1:52-5.

2. Kozlov VI, Bargov SN, Anisimova SY, Opsipov AV. Non-penetrating deep sclerectomy with collagen. Eye Microsurg. 1990;3:44-6

3. Yalvas IS, Sahin M, Eksioglu U, Midillioglu IK, Aslan BS, Duman S. Primary viscocanalostomy versus trabeculectomy for primary open-angle glaucoma: three-year prospective randomized clinical trial. J Cataract Refract Surg. 2004;30(10):2050-7.
4. Shaarawy T, Nguyen C, Schnyder C, Mermoud A. Five year results of viscocanalostomy. Br J Ophthalmol. 2003;87(4):441-5.

5. Stegmann RC, Pienaar A, Miller D. Viscocanalostomy for open-angle glaucoma in black African patients. J Cataract Refract Surg. 1999;25(3):316-22.

6. Neri-Aranguren I, Croxatto O, Grigera DE. Midterm ultrasound biomicroscopy findings in eyes with successful viscocanalostomy. J Cataract Refract Surg. 2002;28(5):752-7.

7. Roters S, Lüke C, Jonescu-Cuypers CP, Engels BF, Jacobi PC, Konen W, Krieglstein GK. Ultrasound biomicroscopy and its value in predicting the long term outcome of viscocanalostomy. Br J Ophthalmol. 2002;86(9):997-1001.

8. Marchini G, Marraffa M, Brunelli C, Morbio R, Bonomi L. Ultrasound biomicroscopy and intraocular-pressure-lowering mechanisms of deep sclerectomy with reticulated hyaluronic acid implant. J Cataract Refract Surg. 2001;27(4):507-17.

9. Park M, Tanito M, Nishikawa M, Chihara E. Ultrasound biomicroscopy of intrascleral lake after viscocanalostomy and cataract surgery. J Glaucoma. 2004;13(6):472-8.

10. Khairy HA, Atta HR, Green FD, van der Hoek J, AzuaraBlanco A. Ultrasound biomicroscopy in deep sclerectomy. Eye (Lond). 2005;19(5):555-60.

11. Chiou AG, Mermoud A, Underdahl JP, Schnyder CC. An ultrasound biomicroscopy study of eyes after deep sclerotomy with collagen implant. Ophthalmology. 1998;105(4):746-50.

\section{Autor correspondente:}

Dr. Lígia Ribeiro

Serviço de Oftalmologia, Centro Hospitalar Vila

Nova de Gaia - Espinho

Rua Conceição Fernandes, no 4434 - 502 Vila Nova

de Gaia, Portugal

Fax: 227868352

E-mail: ligiamfr@gmail.com 